\title{
A Particle Filter Approach to Respiratory Motion Estimation in Nuclear Medicine Imaging
}

\author{
Rahni, A.A.A.; Lewis, E.; Guy, M.J.; Goswami, B.; Wells, K.
}

\begin{abstract}
With the continual improvement in spatial resolution of Nuclear Medicine (NM) scanners, it has become increasingly important to accurately compensate for patient motion during image acquisition. Respiratory motion produced by normal lung ventilation is a major source of artefacts in NM emission imaging that can affect large parts of the abdominal thoracic cavity. As such, a particle filter (PF) is proposed as a powerful method for motion correction in emission imaging which can successfully account for previously unseen motion. This paper explores a basic PF approach and demonstrates that it is possible to estimate temporally non-stationary motion using training data consisting of only a single respiratory cycle. Evaluation using the XCAT phantom suggests that the PF is a highly promising approach, and can appropriately handle the complex data that arises in clinical situations.
\end{abstract}

This paper appears in: Nuclear Science, IEEE Transactions on, Issue Date: Oct. 2011

\section{INTRODUCTION}

NUCLEAR MEDICINE (NM) is the most sensitive approach for imaging functional processes of the human body and is an important tool in oncology, particularly in the early diagnosis of cancerous lesions. There has been significant improvement in spatial image resolution and acquisition time with single photon and positron imaging technologies. However, absolute scan times are still much longer than the single breath-hold period characteristic of X-ray radiology (5-15 minutes for PET, 15-30 minutes for SPECT [1]) and thus scans are still subject to significant patient motion. As spatial resolution improves, it then becomes increasingly important to adequately address the issue of patient motion during such scans.

Motion during NM imaging can be categorized into three broad types: voluntary/involuntary motion, cyclic cardiac motion and respiratory motion. The first of these can be assumed to involve coherent movement of large parts of the body such as the head, limbs and torso, hence it can be described easily and accounted for with an external tracking system such as a 3D optical camera system. In cardiac imaging, cardiac cycle motion is a more challenging problem and proposed solutions involve cardiac gating using ECG devices [2]. For respiratory motion, respiratory gating can also be employed but correction is more challenging due to the complex behavior of the respiratory motion of the 
internal organs. Therefore, it is proposed that applying respiratory motion correction continuously in time may be a more suitable approach and this can be performed for example, on list mode data before reconstruction of the NM images. Proposed solutions for respiratory motion correction include direct registration of NM images [3], [4], [5]. This only requires an additional single breathhold CT image. However, the motion that is detected is highly dependent on the quality of the registration possible with NM images. Other authors propose the inclusion of motion models, which can be constructed from CT [6], [7] or MRI [8]. This approach though has uncertainty on how these models are dependent on the NM or MR images. This leads to the final proposal suggested by a number of authors [9], [10], [11] whereby internal motion is correlated to motion on the external body surface. However, such correlation models are still largely deterministic and are thus constrained by the data on which they are trained.

In acknowledging the complexity and uncertainties involved in respiratory motion, it seems most suitable to use a probabilistic description of the process, which is the basis of the particle filter (PF) approach. To the knowledge of the authors, this represents the first time a PF has been proposed in NM motion correction. Using a PF, organ motion not seen within training data may be correctly accounted for. Section II introduces the PF along with a general description of respiratory motion and the XCAT [12] simulation of respiratory motion used for this initial evaluation of the PF approach. Section III details the implementation of the particle filter. Section IV describes the method of evaluating the PF approach and Section V presents and discusses the results. Finally Section VI closes with the concluding remarks and also includes an outline of prospects for future development of the PF approach in respiratory motion correction.

II BACKGROUND

A. Respiratory Motion

Human respiratory motion is driven mainly by the actions of the ribcage and diaphragm [13]. In normal breathing the

\begin{tabular}{ccc}
\hline \hline \multirow{2}{*}{ Parameter } & \multicolumn{2}{c}{ Respiratory Type } \\
& Normal & Extreme \\
\hline SI motion $(\mathrm{cm})$ & $1-2$ & 10 \\
AP motion $(\mathrm{cm})$ & 1.2 & 3 \\
Rate $(\mathrm{bpm})$ & $12,10-18$ & $34-45,60-70$ \\
\hline \hline
\end{tabular}

TABLE I VARIABILITY IN RESPIRATORY PARAMETERS 


\begin{tabular}{ccc}
\hline \hline Parameter & & Value \\
\hline \multirow{2}{*}{ Body } & Height & $192 \mathrm{~cm}$ \\
\cline { 2 - 3 } & Weight & $95.05 \mathrm{~kg}$ \\
\hline \multirow{2}{*}{ Respiratory motion } & SI & $2.0 \mathrm{~cm}$ \\
\cline { 2 - 3 } & AP & $1.2 \mathrm{~cm}$ \\
\hline \multirow{2}{*}{ Digitization } & Voxel size & $(3.25 \mathrm{~mm})^{3}$ \\
\cline { 2 - 3 } & Frame rate & $2 \mathrm{~s}^{-1}$ \\
\hline \hline
\end{tabular}

\section{TABLE II DEFAULT XCAT PARAMETERS USED}

diaphragm moves around 1-2 cm in the superior-inferior (SI) direction. The general amplitude of this motion can increase to around $10 \mathrm{~cm}$ in deep breathing. On the other hand, the ribcage moves around $1.2 \mathrm{~cm}$ in the anterior-posterior (AP) direction during normal breathing [12]. In deep breathing the amplitude of this motion can increase up to $3 \mathrm{~cm}$ [14]. As a consequence of the movement of the ribcage, the external surface of the chest exhibits similar amounts of movement.

Temporally, the adult human normally performs around 12 breaths per minute [15], [16]. This rate can vary around 10-18 breaths per minute (bpm) [17], [18]. During exercise, the breathing rate can increase to 35-45 bpm. The breathing rates of athletes peak at around 60-70 bpm [19], although in the context of diagnostic imaging, this might only be expected during episodes of hyperventilation. A summary of variability in respiratory parameters based on [12], [13], [14], [15], [16], [17], [18], [19] is presented in Table I.

\section{B. Simulated Data}

The simulation of human respiratory breathing used here is obtained using the XCAT digital phantom. A major advantage in using XCAT is that is allows for tracking of individual points, so that voxel errors can be calculated and quantitatively analysed. In XCAT, organ shapes are modeled using non-rational uniform B-splines (NURBS). The shapes are based on a male and female cadaver of the Visible Human Project CT database.1 Affine transformation of the NURBS control points then simulates respiratory motion. The control points are moved based on respiratory mechanics and respiratory-gated CT data of a normal patient [12].

This paper uses the default XCAT male parameters for normal breathing as shown in Table II. These parameters are used for the training dataset. The variability shown previously in Table I will then be the basis of simulated test breathing sequences used for evaluating the particle filter approach. These test datasets will be described in Section IV. 


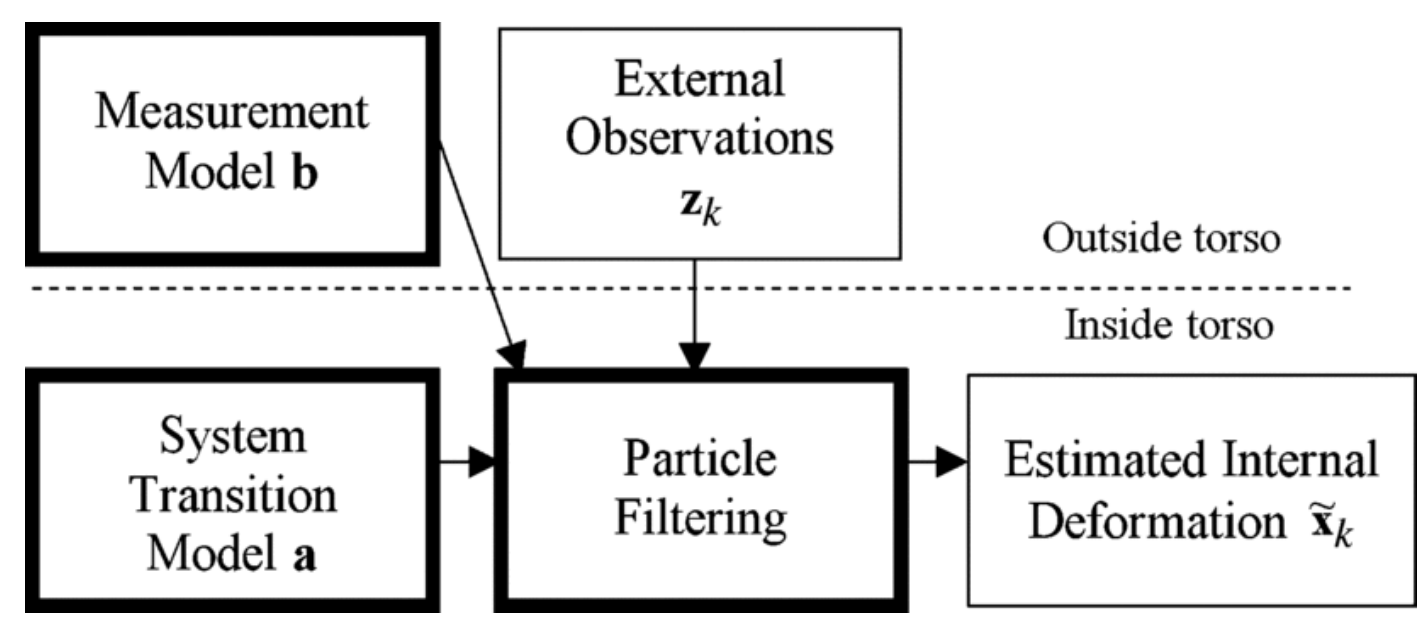

Fig. 1. General outline of the particle filter framework for tracking internal organ deformation for use in respiratory motion correction in NM. The bolded boxes make up the particle filter framework.

C. Particle Filter Framework

Particle filtering is a sequential Monte Carlo estimation technique for Bayesian tracking problems which involve nonlinear functions and/or non-Gaussian probability densities [20]. It has been used in many applications in computer vision and estimation in communication systems [21]. In medical imaging it has been used in image segmentation [22], [23] and registration [24], [25] and in tracking [26], [27], [28]. A particle filter can be used in any application that can be described as a Bayesian tracking problem and it is within this paradigm that respiratory motion estimation is addressed. A general outline of the particle filter approach in this paper is represented in Fig. 1. In this application, the observable, Formula, is the anterior portion of the external torso surface. The hidden state of the system which is to be tracked is denoted Formula, in this case, representing configurations of the internal organs. The PF estimates the current state using the current observation, Formula, and measurement and system transition models, Formula and Formula respectively where Formula represents a discrete time index. These models are based on some prior knowledge.

In Bayesian tracking, which forms the basis of particle filters, the system is described in a state-space form [29]: 
Equation (1) describes how the state, Formula evolves and (2) describes how the current observation, Formula is related to the current state, Formula. The models Formula and Formula have respective stochastic components Formula and Formula to represent uncertainties. Because of the stochastic components, the state and observation can also be represented by their respective probability densities Formula and Formula and the two representations are mathematically equivalent to each other as indicated by the double arrows. The state-space form described by (1) and (2) is illustrated in Fig. 2, showing the system as a first order hidden Markov model.

An estimate of the correct hidden state (i.e. organ configuration) can be deduced from the posterior probability density Formula. This posterior is conditional on the set of all observations Formula. The actual estimate of the state may then be taken as some moment of Formula. In a particle filter, the posterior Formula is approximated as

The approximation in (3) samples probability (indicated by the weighted impulse train) along the space of Formula (i.e. possible states) by a set of Formula point masses, Formula. The point masses are called particles and constitute Monte Carlo samples of the state space. The probability of the posterior at the locations of these point masses are given by the weights, Formula [20], [30]. In this work the expected value Formula is used as an estimate of Formula as this represents a weighted average of all the particles and thus compensates for any error in any particular particle.

In applying this particle filter approach to the problem of respiratory motion correction in NM, it is proposed that the transition and measurement models Formula and Formula are found from a training stage outside of the NM emission process. This stage could for example, consist of a dynamic low dose CT scan to construct the transition model Formula [31], [32], while a simultaneous stereo camera observation of the torso surface is utilized to construct the measurement model Formula. During the actual NM scan, a simultaneous stereo camera capture of the torso surface will again be deployed to provide the observation, Formula [33]. The current configuration of the organs as denoted by the state Formula is then estimated by the particle filter (PF). In practice, such an approach could be implemented on a combined SPECT/CT or PET/CT scanner. It is assumed that the coordinate systems between the two modalities are easily mapped to each other and hence the subject-specific training data is easily mapped onto the coordinate system of the functional emission data. Unlike a purely deterministic approach, wherein marker displacement rigidly determines organ displacement as seen during training, the PF allows for previously unseen marker motion and adapts appropriately. This paper will concentrate on the development and application of the PF in respiratory motion correction in NM. Further details and an overview of how the components of the system function together can be found in [34]. 


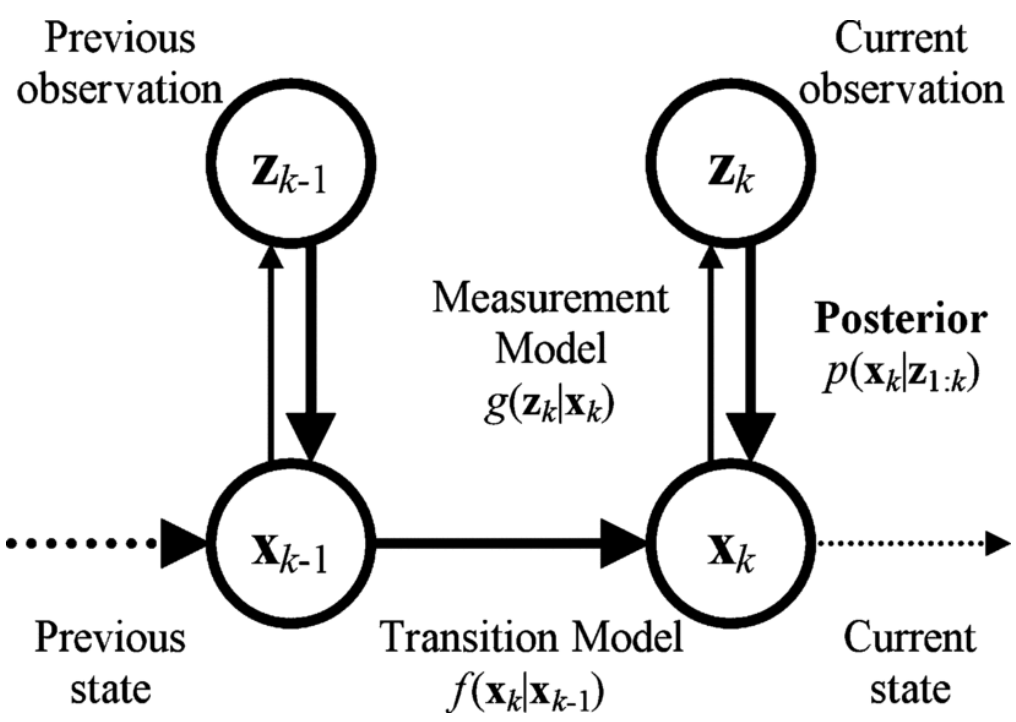

Figure 2

Fig. 2. Diagram of the system in state-space form illustrating the structure as a first order hidden Markov model. The posterior of the current state is represented by the bolded arrows and circles showing the information available at time Formula.

III METHODOLOGY

A. Particle Filter Implementation

The particle filter is implemented as a Sampling Importance Resampling (SIR) filter [20]. In this implementation, the particles or (organ configuration) propositions, Formula are generated through Monte Carlo sampling of the transition density, Formula. Consequently, the weights are then proportional to the measurement density (see Fig. 3):

Equation (4) is implemented by first using an estimate of the measurement density, Formula as an initial value for the weights. The weights are then normalized so that they sum to unity. 


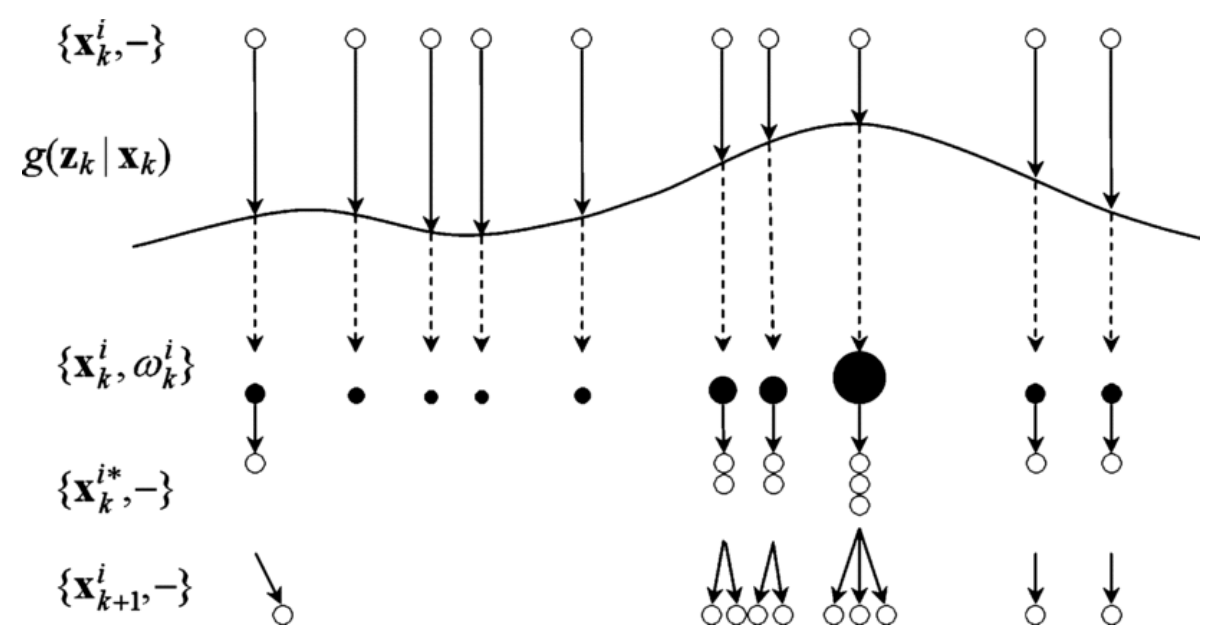

Fig. 3. Illustration of an iteration of the SIR filter. Diagram based on [20]. The column of text on the left is the mathematical representation of objects shown in the illustration (circles and curve). From the top, the position of each particle Formula is used to evaluate Formula, which gives the weights Formula for each particle. Large weights produces more copies Formula, which are propagated to the next time step.

In an SIR filter, resampling from the posterior (3) is also performed at each time step Formula so that the particles are more concentrated at regions where the state is more probable. This is to avoid degeneracy, i.e. the situation where many particles have negligible weight. This is achieved by forming a new set of samples Formula which are related to the original samples, Formula such that

As a consequence of (5), at each time step, particles with larger weights will have more copies that are propagated to the next time step. Particles with very small weights have a low probability of propagation. The resampling step in an SIR filter is thus analogous to how genetic algorithms work. An iteration of the SIR filter is illustrated inFig. 3 and further details can be found in [20].

\section{B. Transition and Measurement Models}

To generate particles from the transition density Formula, its generative form Formula is used, as introduced in (1). As a simple first assumption, a second-order autoregressive process, AR(2), has been chosen as the transition model Formula. It is postulated that this would reasonably represent the pseudo-oscillatory nature of respiratory motion [12] whilst also accounting for stochastic variations in organ configurations at any particular instant. By having the $A R(2)$ process describing the evolution of organ configurations in another variable, Formula, the state Formula can be made to consist of organ configurations from two time points, Formula and Formula. The AR(2) process is thus defined as 
Where now has elements of independent standard normal variables with LO giving the required covariance, Formula is an intercept vector allowing elements of Formula to have non-zero timeaverage values and Formula are the AR matrices. The parameters for 6 are found from stepwise least squares (LS) estimation [35] on known training data (e.g. the proposed low dose dynamic CT data [31], [32]). Equation (6) can be written as a first-order autoregressive process, $A R(1)$, in terms of the full state vector, Formula. Hence it can be seen that Formula (Fig. 2) is a first-order Gaussian-Markov process [30]:

For initial evaluation, the measurement density Formula is also assumed to be Gaussian. Its generative form Formula is thus a linear map of the state with a stochastic component:

where is defined as in (7) and consists of organ configuration from two adjacent time points. Formula in 8 also has elements of independent standard normal variables resulting in the noise covariance to be Formula. This covariance accounts for estimated inaccuracy of the map constants (Formula and Formula) and observation noise. In this paper the noise is assumed to be isotropic with a root mean square error (RMSE) of $0.25 \mathrm{~mm}$ based on the Polaris2 3D optical stereo tracking system. This system has had popular application for motion correction in medical imaging and treatment [2]. Similar to (6), the coefficients of (8) are also found using LS regression on training data.

\section{Tracked State}

PF applications often track particular objects (e.g. in [26], [27], [28] and [30]). However, in this paper, Formula consists of affine transformation parameters for each organ Formula at each time point Formula for registration back to a baseline configuration Formula, selected from training data. Thus for the 3D coordinates of the set of Formula voxels of organ Formula at time Formula, Formula, the following affine transformation is assumed to approximate deformation due to respiratory motion:

In (9), Formula accounts for scale, shear and rotation whereas Formula accounts for translation. Their elements for all selected organs together constitute Formula. The selected organs are the heart, liver, spleen, kidneys, lungs and ribs. Surface renders of the chosen organs generated from XCAT are shown in Fig. 4. The transformation parameters in (9) for the training stage are found using Iterative Closest Points (ICP) registration. Implementation details of the registration stage can be found in [32]. For the purpose of this paper, the baseline configuration Formula is taken to be the respiratory rest phase in XCAT, which is at maximum exhalation. 


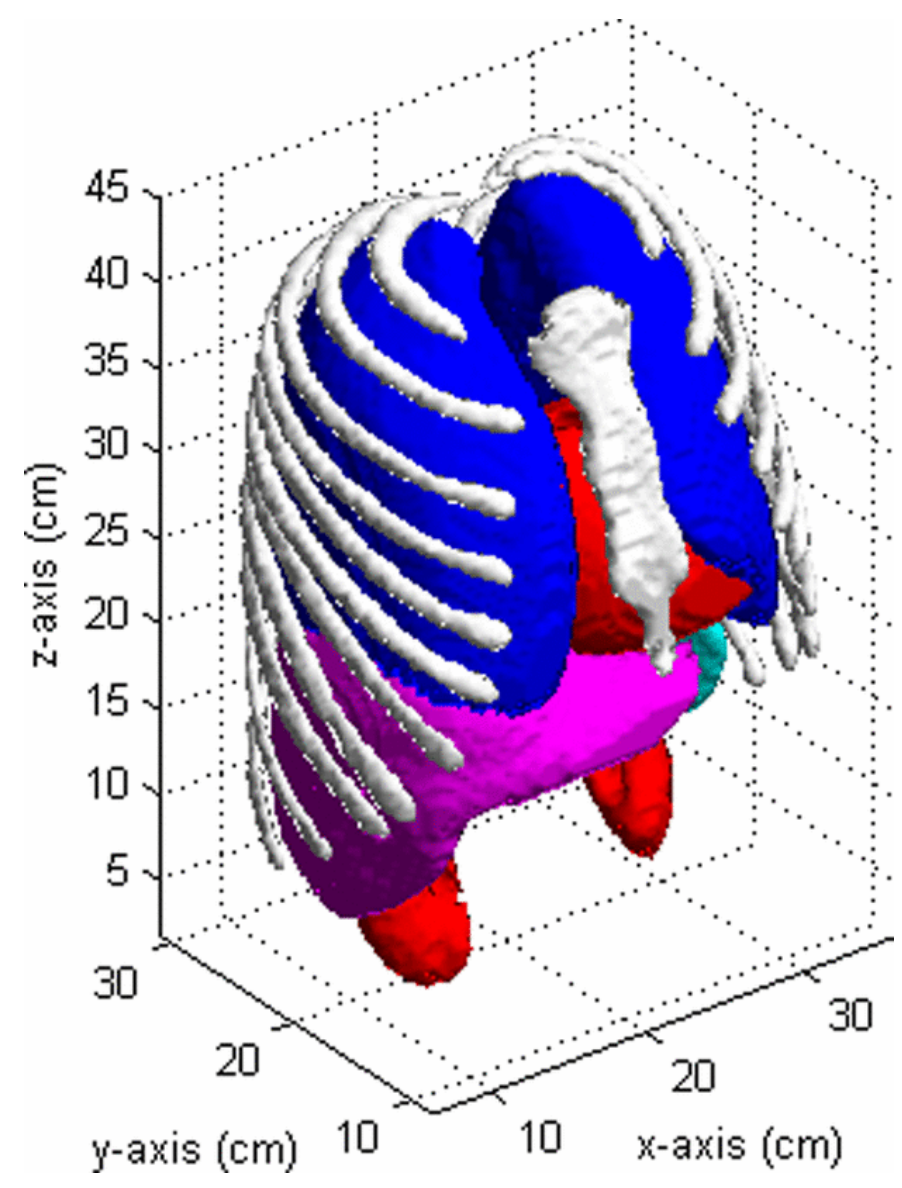

Fig. 4. Surface renders of the chosen organs.

D. Novel Adaptations

In addition to the tracking of registration parameters rather than physical objects, several application specific adaptations have been made to the PF. In the current implementation of the PF, exploration of the state space may be deficient. This is because the SIR filter intrinsically uses a suboptimal importance density i.e. the propagation density Formula, and because simplistic transition (6) and measurement (8) models are used. Furthermore, as the training stage of the particle filter framework is assumed to consist of known organ configurations representing only one normal respiratory cycle, the transition and measurement models have a tendency to be over fitted to training data and thus their stochastic components will not reflect the actual variability seen during the NM imaging process.

Therefore, three methods have been employed so that the models used in the PF framework can adapt to the expected variability in natural respiration. These methods are: 
Dimensionality reduction,

Incorporation of estimated respiratory parameters, and

Planned sampling of particles.

The process flow of how the adaptation methods fit into the training and test stages of the PF framework is illustrated in Fig. 5. The adaptation methods are described in more detail in Sections III-D-1to III-D-3 that follow:

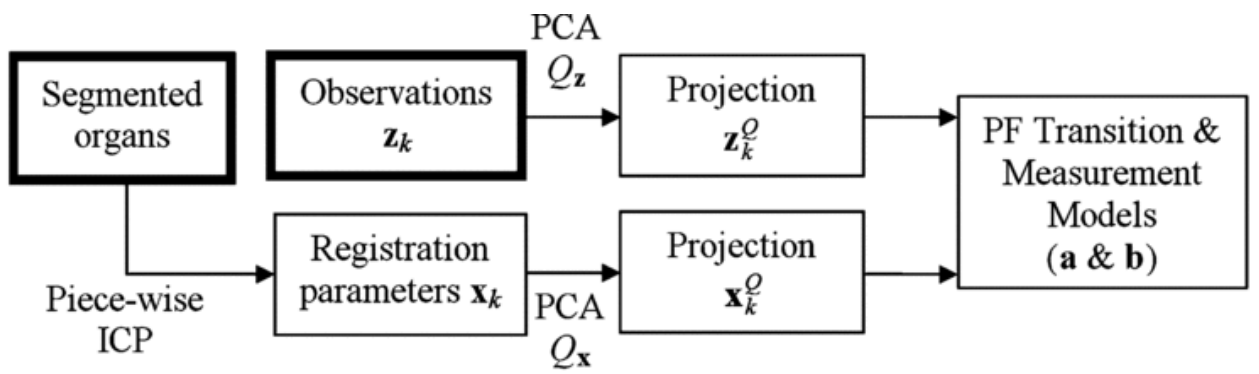

(a)

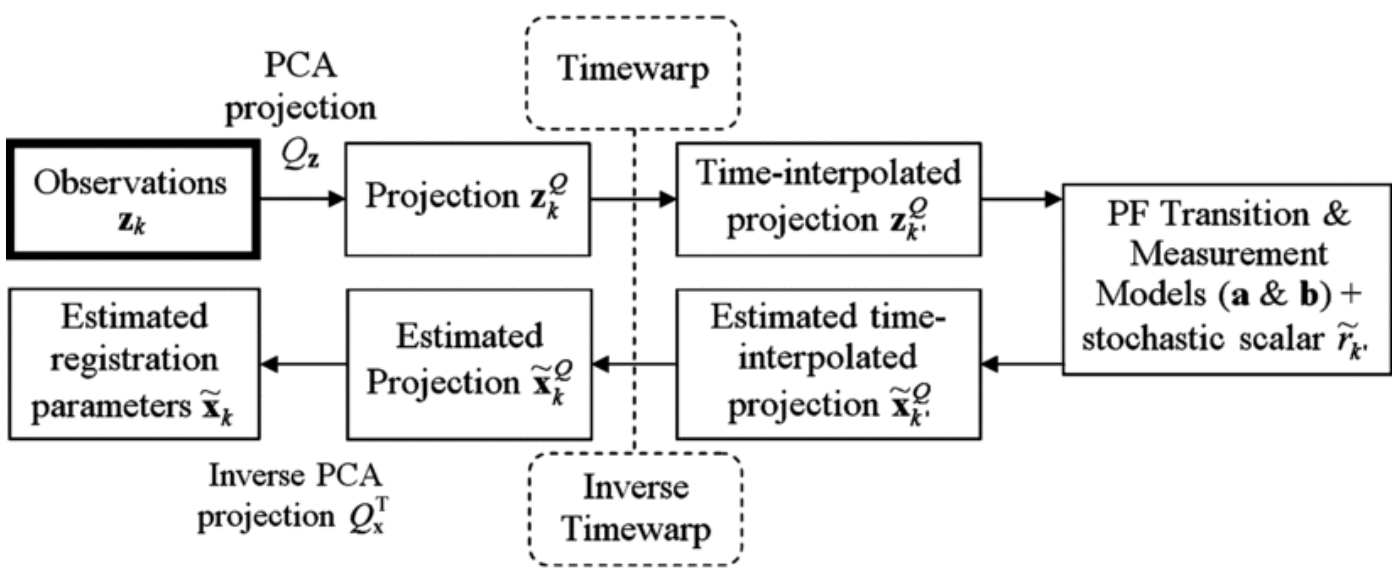

(b)

Fig. 5. Diagrams show how the adaptation methods fit into the PF framework. Dimensionality reduction is implemented as PCA projections Formula and Formula, estimated respiratory parameters are included as the timewarp and stochastic scalar Formula. Formula is then used in planned sampling of particles. (a) Detailed illustration of the training stage of PF models Formula and Formula. The bolded boxes represent data available during training. (b) Detailed illustration of how the PF models Formula and Formula are used to produce the estimated registration parameters Formula, using the current observation Formula.

\section{1) Dimensionality Reduction}

As the transition 6 and measurement 8 models tend to be over fitted to training data, the high dimensionality of the state requires an exponentially increasing number of particles in order for adequate sampling of the state-space [36]. Therefore principal component analysis (PCA) is used to reduce the dimensionality of the state-space. PCA is performed using compact singular value 
decomposition, so that the number of principal components (PCs) that are kept is based on the rank of the training data matrix. The maximum number of PCs is therefore equal to the number of data points in the training stage, as the discarded principle axes have eigenvalues of zero [37].

Using PCA, the dimensionality of state and observation vectors in the transition 6 and measurement 8 models are decreased. The projected state, Formula is obtained from the state, Formula as follows:

has rows corresponding to the chosen PCs. Formula is an assumed mean value of the state. This mean value is taken to be zero except for the affine scale parameters, where their mean is assumed to be 1 . As Formula is orthonormal, the state can be obtained from its projection as follows:

When choosing the number of PCs to be included for the transition model 6 , one criteria is that the variances of each of the included PCs are not too small that they affect LS estimation of the AR(2) parameters through ill-conditioned matrices. As such, PCs which have log-eigenvalues below a threshold found by Otsu's method [38] are excluded. Additionally, the variance of the first PC of the projected state, Formula, is also increased by the amount given by an $A R(2)$ approximation using only the first PC.

Similar to finding the projection of the state to PCA space in (10) and inverse projecting in (11), the projection of the observation, Formula is also found using a projection matrix $Q z$ which has rows corresponding to the chosen PCs for the observation. Consequently the inverse projection is found using Formula. The mean value for the observation is taken to be those at the rest phase. The number of PCs chosen for the observation is set to be equal or more than the number of PCs chosen for the state. This is so that the measurement model does not represent an under determined system of equations.

\section{2) Incorporation of Estimated Respiratory Parameters}

The respiratory parameters which are estimated are the cycle period and amplitude ratio i.e. the ratio of the amplitude relative to that of the training cycle. These parameters are calculated from the same PCA of the observation that is used in adaptation method 1. The largest principal component, Formula is projected from observation data as follows:

FormulaTeX Source where Formula is the principle direction and Formula is the observation data matrix for Formula observations, with 
Formula is an assumed time-average value which is taken to be the coordinates of the observation points at the rest phase. The deflection points of this principle component are then used to estimate the respiratory period and amplitude between each deflection point.

The estimated respiratory parameters are used in two ways:

Time-warping observation data so that each half-cycle matches the period of the training data. This is accomplished using cubic spline interpolation.

The estimated amplitudes of each half-cycle are used as scaling factors in planned sampling. This is designated as adaptation method 3.

3) Planned Sampling of Particles

In planned sampling, the samples or particles Formula are modified at every time point when respiration is estimated to be at the rest phase. This method of planned sampling is similar to that introduced in [39]. However, in the PF framework of this paper, the sampling of all particles is planned rather than just a proportion of them. In this paper the planned samples are also derived from training data.

The planned samples use a scale factor based on the estimated amplitude ratio Formula of the current half-cycle. This ratio is calculated from the estimated amplitude of the half-cycles in adaptation method 2. The ratio is defined as the amplitude of a half-cycle relative to that of the training data as measured along the first PC vector. The samples are then generated in two different ways:

At time 1 of each test dataset, the particles are generated by a scaled version of the transition model:

with Formula being propagated from training data.

At other estimated rest phase points, the particles from the previous time step, Formula are simply scaled by a scalar Formula, which is the estimated amplitude ratio of the upcoming cycle with that of the previous cycle.

In both ways of generating the planned samples, the value of the scale factor for every particle has a stochastic component based on the uncertainty in determining the rest phase points. For instance at an estimate rest phase time point Formula:

where is a modified Gaussian scalar with a standard deviation derived from the maximum error in Formula if the rest phase time point was in error by 1 frame of the observable. Samples from a Gaussian that are below -1 are replaced so that the minimum possible value of is 0 . Note that the 
inclusion of this stochastic scale factor effectively prohibits the use of a Kalman Filter for tracking, as the transition model is no longer linear-Gaussian with this modification [20].

\section{SECTION IVEVALUATION}

The application of the PF framework to respiratory motion correction in NM has been evaluated as follows: seven XCAT respiratory cycles have been generated, each representing a dataset with unique set of respiratory parameters. These are outlined in Table III. The first dataset (Test 0 ), is considered to be a training set used to estimate model parameters. In practice this training set might, for example, be derived from low-dose dynamic CT. The remaining datasets have varied respiratory parameters to represent some aspect of natural variation in respiratory motion, as seen in tracking volunteer marker data [14], [33].

\begin{tabular}{lccc}
\hline \hline \multirow{2}{*}{ Dataset } & \multicolumn{2}{c}{ Motion Amplitude (cm) } & \multirow{2}{*}{ Cycle Period (s) } \\
\cline { 2 - 3 } & Diaphragm (SI) & Chest (AP) & \\
\hline Test 0 & 2 & 1.2 & 5 \\
Test 1 & 1 & 0.6 & 5 \\
Test 2 & 3 & 1.8 & 5 \\
Test 3 & 4 & 2.4 & 5 \\
Test 4 & 1 & 0.6 & 3 \\
Test 5 & 2 & 1.2 & 3 \\
Test 6 & 3 & 1.8 & 3 \\
\hline \hline
\end{tabular}

TABLE III VARIABILITY IN RESPIRATORY PARAMETERS

In setting respiratory parameters for the test dataset, the range of respiratory cycle periods is chosen so that it spans the range of respiratory rates during normal tidal breathing, from 12 to 20 breaths per minute. The amplitude is chosen to span the range from half the normal depth of respiration to 1.5 or 2 times the normal amplitude. The upper limit is dependent on respiratory rate as XCAT sets a smaller upper limit when the rate is higher. In order to demonstrate the effectiveness of the PF approach to changes in respiratory motion and to compensate for the fact that XCAT is used for evaluation, the variations that have been deliberately included within the test data are larger than normally expected within a similar period of an actual patient study. The respiratory parameters for the training dataset are also used i.e. as a selftest, albeit with different noise realizations which appears as Test 0 in Table III. In all tests, the respiratory cycles are set to begin at the rest phase i.e. at the end of exhalation, to simplify the error analysis. In reality when respiratory cycles are out of phase, the start phase can be estimated from adaptation method 2 as described in Section III-D.

Evaluation was performed by comparison with XCAT ground truth displacement data. The XCAT phantom environment facilitates the ability to track the position of individual voxels, and thus errors associated with any particular correction scheme can be evaluated. In this application XCAT provides the known position of a 3D grid of organ-labelled points throughout a respiratory cycle. 
Observation points or markers on the anterior torso surface are used to produce estimates of organ configuration in each test case. The observable, Formula is chosen to be a grid of 3D points on the anterior portion of the external surface of the torso. Projections of the points onto the coronal plane are spaced $7.8 \mathrm{~cm}$ apart from each other. Fig. 6 shows the position of these points. In practice the observable might correspond to physical markers.

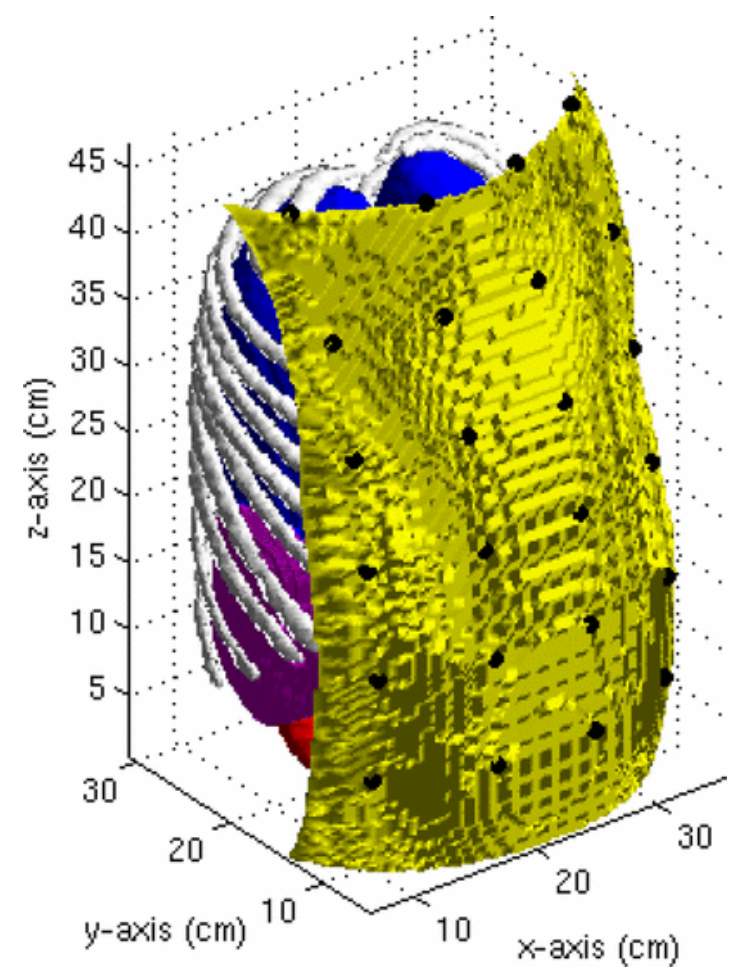

Fig. 6. Surface render of an anterior portion of the external surface of the torso with chosen organs rendered as in Fig. 4. The points chosen as the observable are marked as circles Formula.

XCAT data has been simulated at 2 frames/second to represent a reasonable approximation to how dynamic low-dose CT data can be binned temporally. The camera data would in practice, operate at around 10 times higher frame rate. However, simulating XCAT at such a high rate would provide unrealistically high sampling in the corresponding organ positions. Therefore observation data is interpolated in time using cubic splines to simulate an observation rate of 6 frames/sec.

White Gaussian noise is also added to the observation data with an isotropic standard deviation of $0.25 \mathrm{~mm}$ to represent uncertainty in the simulated stereo camera observation This noise is added to all observations in the training and test dataset and a plot of relative motion in the spatial axes of a single point from the observation data is illustrated by Fig. 7 . 


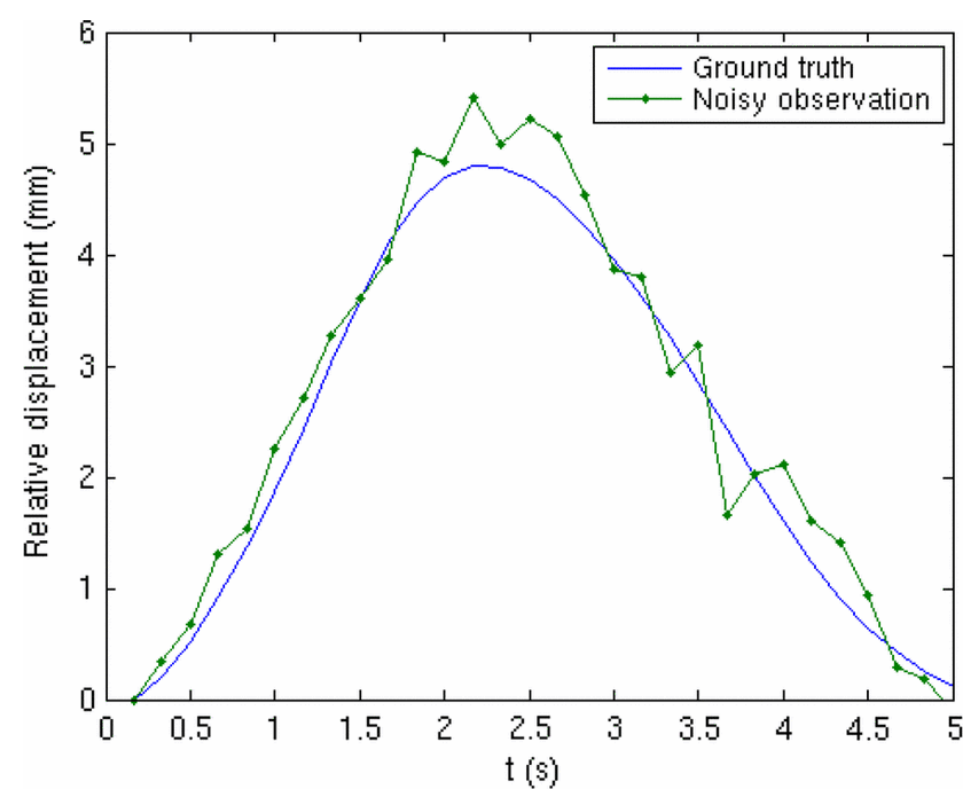

Fig. 7. Plot of relative displacement for one chosen point of the observable of the training dataset. The deviated displacement of the observable caused by simulated noise is contrasted with the ground truth from XCAT.

To evaluate the accuracy of organ registration to a reference frame Formula, another grid is initialized to voxel coordinates of that reference frame. The position of these points on the grid during test sequences are then transformed in an affine manner using parameters estimated by the particle filter approach for the selected organ and compared to the positions in the reference frame. The particle filter (PF) framework is applied to the test datasets using the application specific adaptations, as described in Section III-D. The results and discussion now follow in Section V.

\section{RESULTS AND DISCUSSION}

Using the test datasets outlined in Table III the Euclidean distance error averaged over organ voxels is shown in Fig. 8. The error for the test sequences are plotted sequentially with the rest phase (maximum exhalation) as the reference frame Formula at time 0 . Each respiratory cycle represents one of the test datasets. The result for test 0 starts at the rest phase, whereas all other tests start at the next frame. The results for each test stops at one frame before the length of their cycle period because the next frame is simply the rest phase. In Fig. 8 it can be clearly seen that the errors for the major organs are extremely low, but somewhat erratic over time. The largest errors occur in the lungs. The errors for the ribcage follow a similar temporal pattern to that of the lungs but are of lower magnitude. 


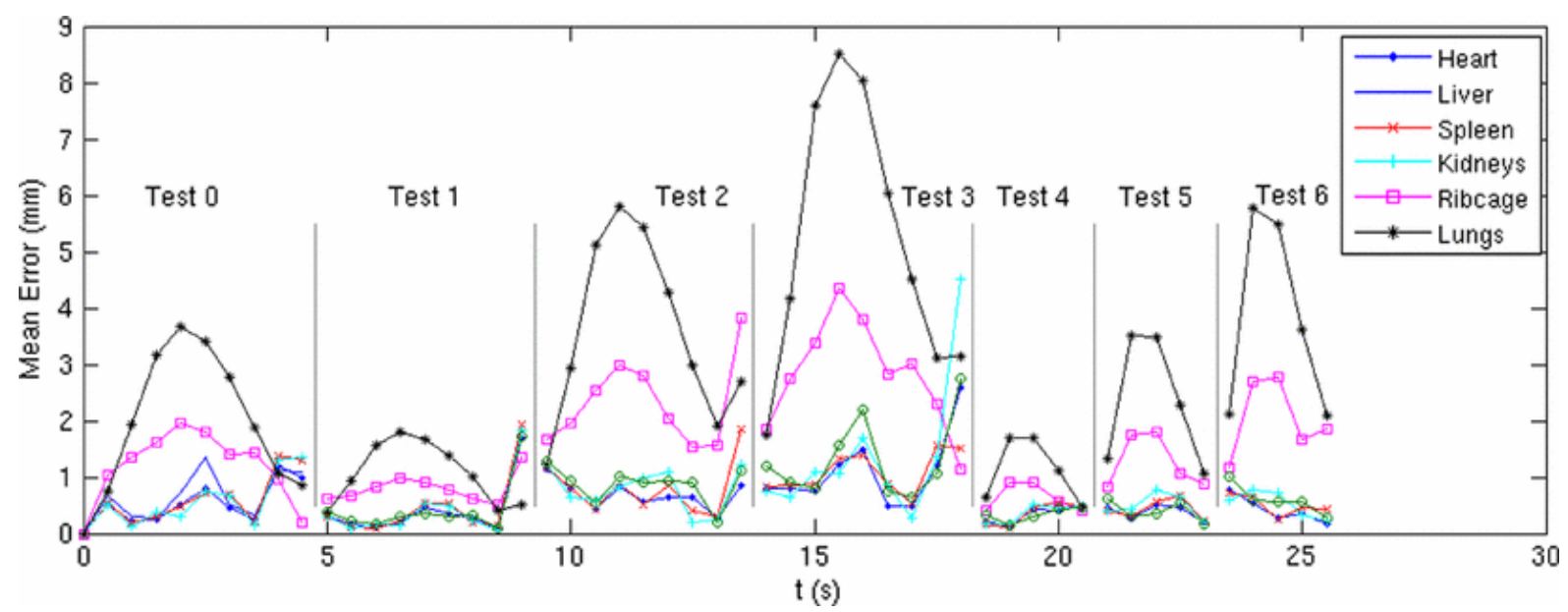

Fig. 8. Mean Euclidean distance errors for the PF shown for each organ. The error sequences are plotted sequentially with the reference frame Formula at time 0.

Fig. 9(a) plots the errors averaged over each test dataset against relative amplitude (to that of training data) for respiration with cycle periods that match that of the training dataset (5 s). From this plot it is evident that a large factor affecting the errors for the lungs and ribcage is the respiratory cycle amplitude. Similar to the errors for the lungs, the errors for the ribcage increase with the amplitude ratio (relative to the training dataset), but the trend has a smaller gradient. This dependence on amplitude may be due to under compensation of the respiratory motion. Hence in respiration with lower cycle amplitudes there is less compensation to be performed than in respiration with higher cycle amplitudes. The errors for the other organs are smaller than the errors for the ribcage, with a slight dependence on amplitude but the gradient somewhat decreases with respiratory amplitude. In Fig. 9(b), when the period is reduced to $3 \mathrm{~s}$, the errors produced are very similar to when the period is $5 \mathrm{~s}$ and are only on average $0.1 \%$ larger. There is no test dataset where the relative amplitude equals two and Formula as explained in Section IV. 


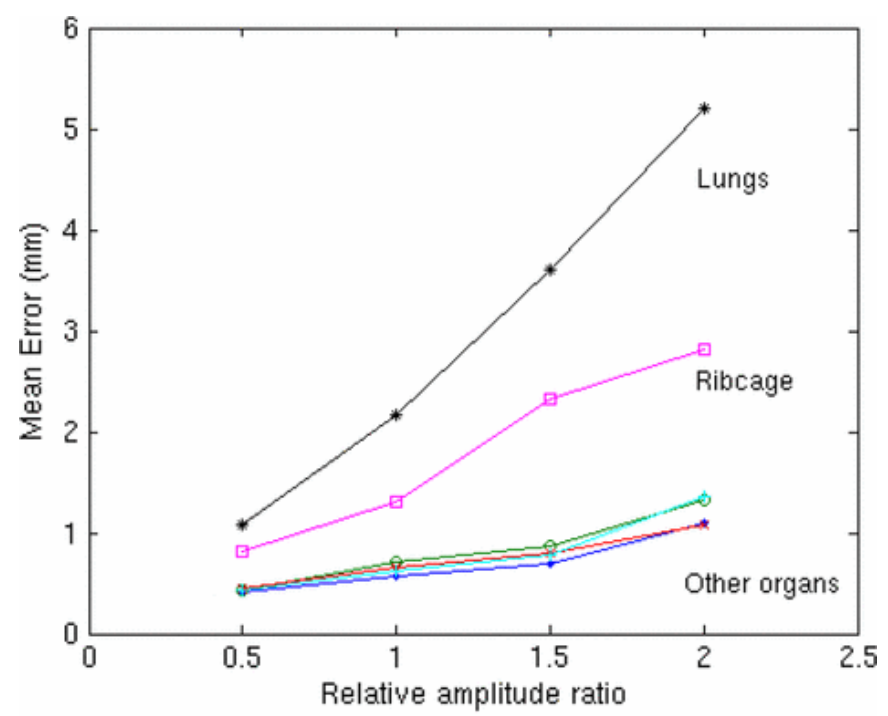

(a)

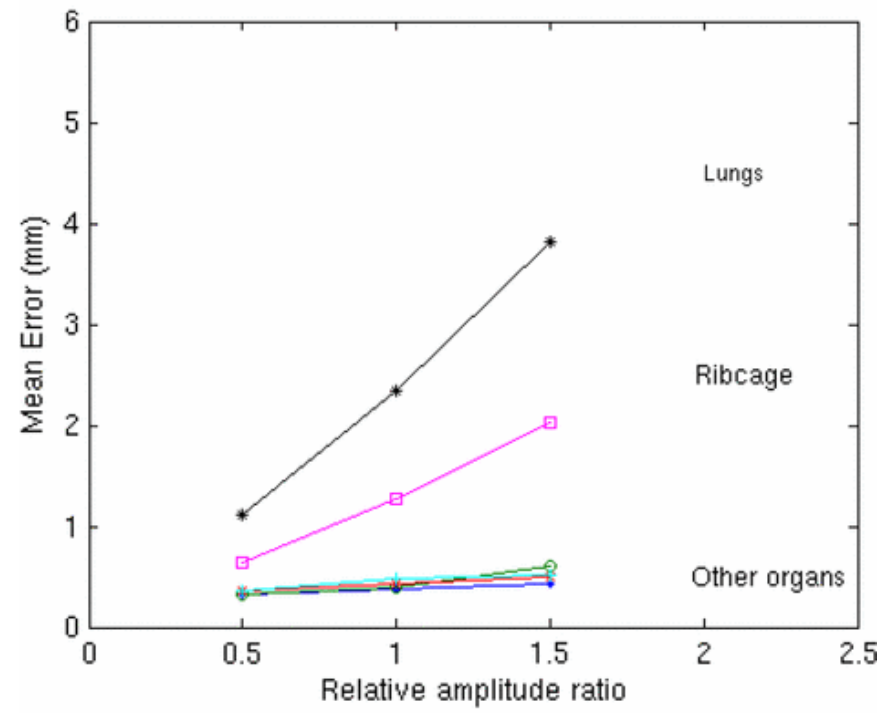

(b)

Fig. 9. Mean error $(\mathrm{mm})$ against relative amplitude ratio. Legend follows Fig. 8.

Fig. 10 plots the errors against a cyclic phase index. The phase index runs from 0 to 10 , with 0 being the rest phase and 10 corresponding to phase index 0 . The datapoints at non-integer values of phase correspond to some datapoints from tests 4-6, when Formula. Their phases are inferred from ground truth data. The errors are also averaged across the test datasets with respect to a particular phase index. From this plot it can be seen that the largest errors for the lungs occur at mid-cycle, during maximum inspiration when the organs are furthest away from their rest configuration. The error for the ribcage also follows a similar pattern but with a lower overall magnitude. The other organs again have errors of lower magnitude than that of the ribcage, but do not exhibit any clear trend. 


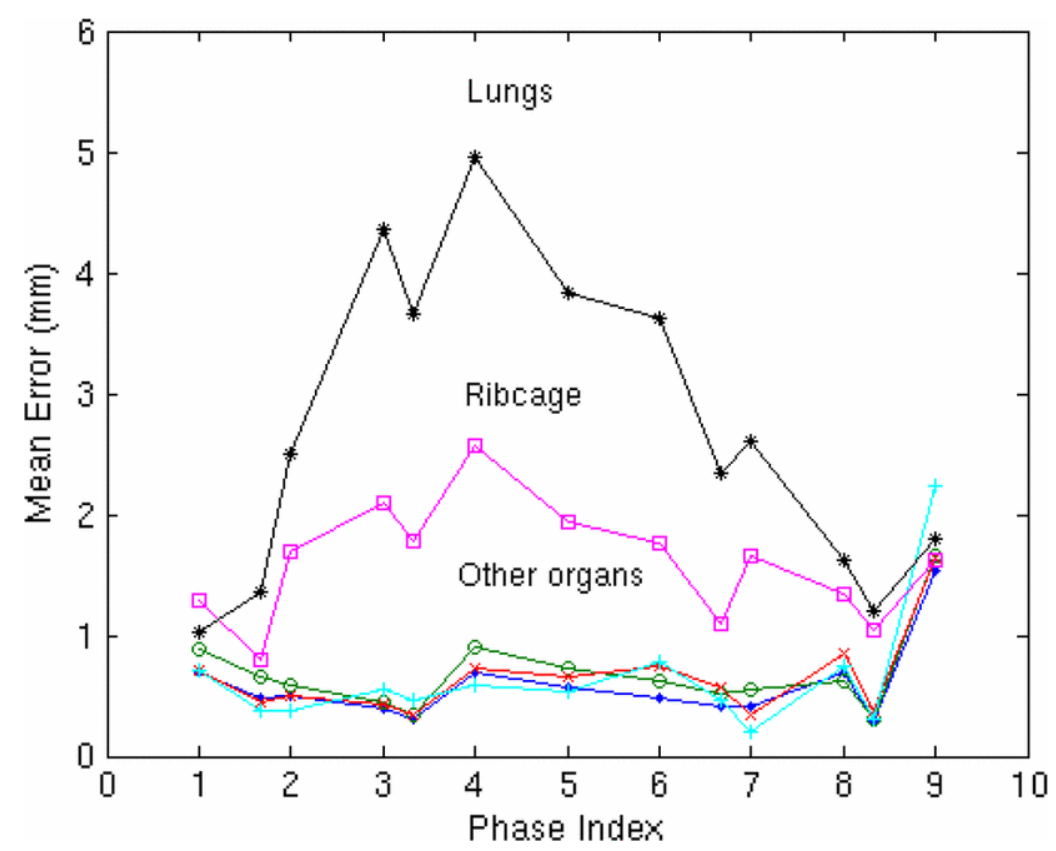

Fig. 10. Mean error (mm) against phase index (0-10). Legend follows Fig. 8.

Fig. 11 shows the errors averaged for each organ, over time and over all test datasets. The error bars show variance over time and over all test datasets. The comparison with the mean errors when there is no motion estimation show that the PF approach has dramatically reduced the effect of respiratory motion. The overall mean error has been reduced from $12.1 \mathrm{~mm}$ to only $1.7 \mathrm{~mm}$ which is almost half the voxel dimension used here $(3.25 \mathrm{~mm})$.

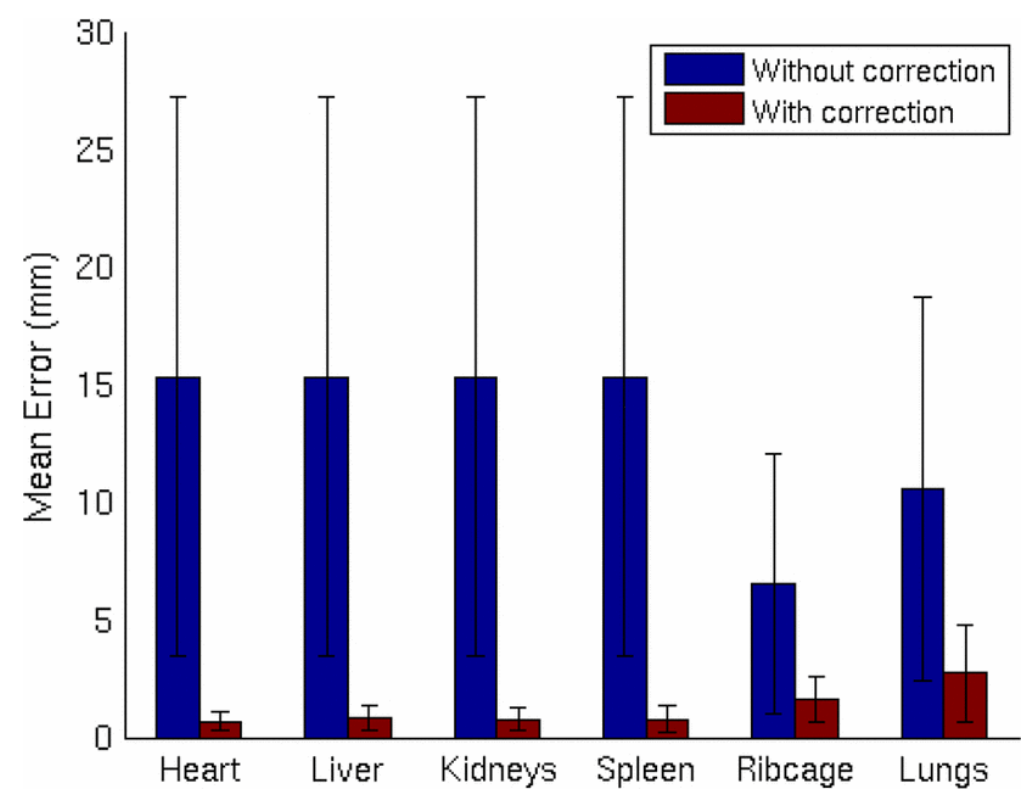

Fig. 11. Mean error caused by respiratory motion, with and without correction by the PF framework. 
Fig. 12 plots the same mean errors using a vertical axis over a more limited range to demonstrate the PF error against the registration error. This shows that overall, the largest errors are at the lungs followed by errors at the ribcage. The average errors for the other organs are just under $1 \mathrm{~mm}$. To investigate this further; errors arising from the registration procedure have been analysed. The ICP registration errors are also shown for comparison, averaged over all the datasets in Table III using the normally hidden configurations of internal organs. The ICP registration error refers to mean voxel error between the actual organ position, and that where ICP estimates it to be. In this regard, it represents the error associated with formulating the state space as a piece-wise affine transformation. This error that is reported is only possible with access to ground truth NM resolution volumes of the test datasets. This is not available to the PF framework. It can once again, be seen that the largest errors for ICP are those for the lungs, followed by the ribcage. The fact that the PF represents organ configurations as affine registration parameters trained using ICP also presents a limit to the accuracy that can be achieved here in using ICP within a PF framework for the data under consideration. Thus the PF framework appears to be able to estimate the state of internal organs accurately, with an overall mean error of $1.7 \mathrm{~mm}$. Any additional error due to the PF is small.

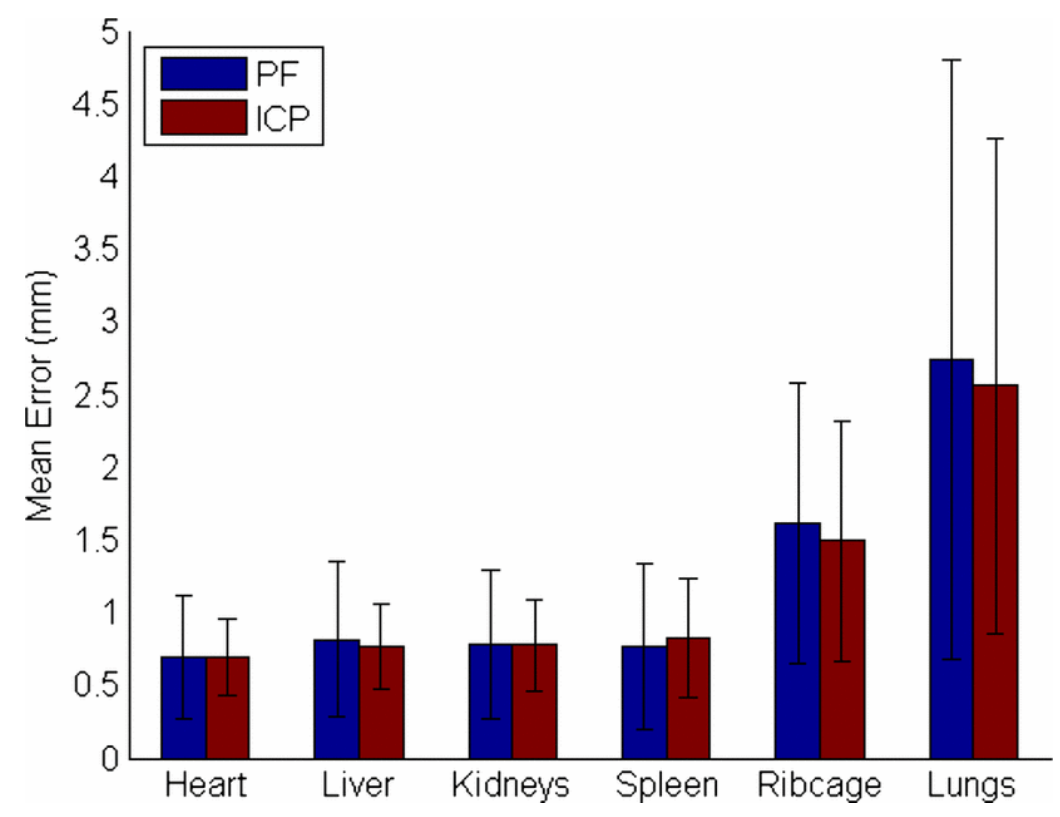

Fig. 12. Mean error after motion estimation using the PF and ICP registration.

Consequently, the error on the PF estimate is dominated by the error associated with the piecewise ICP registration. The linear models in the PF are adequate for this initial test of the motion correction framework. However it is encouraging to note that despite choosing respiratory parameters that consider a wider variation than one might expect to see in a single subject, the PF has been successful at adapting and accounting for this previously unseen motion. It is proposed that the results demonstrated here suggest great potential of the PF framework for successful correction of respiratory motion in NM. 


\section{CONCLUSION AND FURTHER WORK}

The results presented and analysed here show that the PF framework as implemented in this work is a promising method of estimating internal organ state for motion correction in nuclear medicine imaging. The current implementation approximates the transformation needed to account for motion to a sufficient degree of accuracy for use in functional imaging.

There is still scope for improvement based on further development of the piecewise-affine transformation for organ deformation based on the larger errors for the lungs and ribcage. This is the subject of on-going development. However preliminary work with a sample 4D CT dataset suggests that a piecewise-affine transformation is still a reasonable assumption for the real motion of organs [40].

It is also acknowledged that XCAT does not fully simulate the manner of real respiratory motion within a single respiratory cycle. It is believed that by having the tracked state Formula to consist of organ configurations from two time points ((6)), it already alleviates some issues such as hysteresis and unsymmetrical respiratory cycles. There is ongoing work to evaluate whether respiratory irregularities are to be filtered out before observations are passed onto the PF or let the PF handle such irregularities with the inclusion of improved models. A better model may be found using kernel density estimation as have been demonstrated in other work in this research group on respiratory motion prediction of real volunteers [14]. The ability of the PF to handle irregularities can thus be evaluated by combining real respiratory traces with clinical 4D CT data as well as XCAT which is also a topic of on-going work.

Authors

A. A. Abd. Rahni was supported by the Malaysian Ministry of Higher Education and Universiti Kebangsaan Malaysia.

A. A. Abd. Rahni is with the Universiti Kebangsaan Malaysia, 43600 UKM Bangi, Selangor, Malaysia and also with the Centre for Vision, Speech and Signal Processing (CVSSP), Faculty of Engineering and Physical Sciences, University of Surrey, Guildford GU27XH, U.K. (e-mail: a.abdrahni@surrey.ac.uk).

E. Lewis and B. Goswami are with the CVSSP, Faculty of Engineering and Physical Sciences, University of Surrey, Guildford GU27XH, U.K. (e-mail: e.lewis@surrey.ac.uk; b.goswami@surrey.ac.uk).

M. J. Guy is with the Medical Physics Department, Southampton University Hospital Trust, Southampton SO16 6YD, U.K. (e-mail: matthew.guy@nhs.net). 
K. Wells is with the CVSSP, Faculty of Engineering and Physical Sciences, University of Surrey, Guildford GU27XH, U.K. and also with the Department of Radiological Sciences, College of Applied Medical Science, King Saud University, Riyadh 11433, Kingdom of Saudi Arabia (e-mail: k.wells@surrey.ac.uk).

\section{REFERENCES}

1. M. Lodge , H. Braess , F. Mahmoud, J. Suh , N. Englar , S. Geyser-Stoops , J. Jenkins , S. Bacharach and V. Dilsizian "Developments in nuclear cardiology, transition from single photon emission computed tomography to positron emission tomography/computed tomography" J Invas. Cardiol., vol. 17, no. 9, pp. 491-496, 2005,

2. A. Rahmim , O. Rousset and H. Zaidi "Strategies for motion tracking and correction in PET" Posit. Emiss. Tomogr. Clin., vol. 2, no. 2, pp. 251-266, 2007

3. M. Dawood , F. Büther , L. Stegger , X. Jiang and K. P. Schäfers "Respiratory motion correction in 3D PET data with advanced optical flow algorithms" IEEE Trans. Med. Imag., vol. 27, no. 8, pp. 11641175,2008 ,

4. R. G. Wells , T. D. Ruddy , R. A. DeKemp , J. N. DaSilva and R. S. Beanlands "Single-phase CT aligned to gated PET for respiratory motion correction in cardiac PET/CT"

J. Nucl. Med., vol. 51, no. 8, pp. 1182-1190, 2010

5. W. Bai and M. Brady "Motion correction and attenuation correction for respiratory gated PET images" IEEE Trans. Med. Imag., vol. 5, no. 2, pp. 341-353, 2011

6. T. Li , B. Thorndyke, E. Schreibmann , Y. Yong and L. Xing "Model-based image reconstruction for four-dimensional PET" Med. Phys., vol. 33, no. 5, pp. 1288-1298, 2006

7. S. J. McQuaid, T. Lambroud, V. J. Cunningham, V. Bettinardi and N. C. G. B. F. Hutton "The application of a statistical shape model to diaphragm tracking in respiratory-gated cardiac PET images" Proc. IEEE, vol. 97, no. 12, pp. 2039-2052, 2009

8. M. Reyes , G. Malandain , P. M. Koulibaly , M. A. González-Ballester and J. Darcourt "Model-based respiratory motion compensation for emission tomography image reconstruction" Phys. Med. Biol., vol. 55, no. 5, pp. 251-266, 2007

9. R. Boutchko, B. Reutter , D. Saloner and G. Gullberg "Correlating motion of internal organs with the displacements of fiducial markers during respiration" Proc. IEEE Nuclear Science Symp./Medical Imaging Conf. Rec., pp. 3641-3642, 2008

10. M. King , J. Dey , K. Johnson , P. Dasari , J. M. Mukherjee , J. E. McNamara , P. H. Pretorius , A. Konik, S. Zheng and S. Miro"Use of MRI to assess the prediction of heart motion by stereo-tracking of markers on the body surface" Proc. IEEE Nuclear Science Symp./Medical Imaging Conf. Rec., pp. $3320-3325,2010$ 
11. H. Fayad , T. Pan , N. Albargach, C. Roux and D. Visvikis "A generic respiratory motion model for motion correction in PET/CT" Proc. IEEE Nuclear Science Symp./Medical Imaging Conf. Rec., pp. $2455-2458,2010$

12. W. Segars Development of a New Dynamic Nurbs-Based Cardiac-Torso (NCAT) Phantom 2001

13. P. Macklem and J. Mead Handbook of Physiology: Section 3: The Respiratory System; vol. 3, 1986, American Physiological Soc.

14. M. Alnowami , E. Lewis, M. Guy and K. Wells "Respiratory motion modelling and prediction using probability density estimation" Proc. IEEE Nuclear Science Symp./Medical Imaging Conf. Rec., pp. 2465-2469, 2010

15. L. Sherwood Fundamentals of Physiology: A Human Perspective 2005, Thomson Brooks/Cole

16. G. Tortora and N. Anagnostakos; Principles of Anatomy and Physiology 1990, Harper-Collins

17. B. Beckett Illustrated Human and Social Biology 1995, Oxford Univ. Press

18. A. Chandrasekhar; Screening Physical Exam 1998

19. W. McArdle , F. Katch and V. Katch; Exercise Physiology: Energy, Nutrition, and Human Performance; 1996, Williams \&amp; Wilkins

20. B. Ristic , S. Arulampalam and N. Gordon Beyond the Kalman Filter: Particle Filters for Tracking Applications; 2004, Artech House

21. A. P. Petropulu "Special issue on Monte Carlo methods for statistical signal processing" IEEE Trans Signal Process., vol. 50, no. 2, 2002

22. D. Angelova and L. Mihaylova "Fast image reconstruction for Compton camera using stochastic origin ensemble approach" Mach. Vis. Appl., vol. 22, no. 3, pp. 551-561, 2010

23. H. Shim , D. Kwon, I. D. Yun and S. U. Lee "Robust segmentation of cerebral arterial segments by a sequential Monte Carlo method: Particle filtering" Comput. Methods Progr. Biomed., vol. 84, no. 2\&3, pp. 135-145, 2006

24. C. Florin , J. Williams, A. Khamene and N. Paragios "Registration of 3D angiographic and X-ray images using Sequential Monte Carlo sampling" Proc. Computer Vision for Biomedical Image Applications, pp. 427-436, 2005

25. F. Deligianni , A. J. Chung and G. Yang "Nonrigid 2D-3D Registration with catheter tip EM tracking for patient specific bronchoscope simulation" Proc. Medical Image Computing and ComputerAssisted Intervention (MICCAI), pp. 281-288, 2006

26. I. Gergel , T. R. dos Santos, R. Tetzlaff, L. Maier-Hein , H. Meinzer and I. Wegner "Particle filtering for respiratory motion compensation during navigated bronchscopy" Proc. SPIE Medical Imaging 2010: Visualization, Image-Guided Procedures, and Modeling 
27. X. Luó , Y. Hasegawa, K. Mori , M. Feuerstein , T. Kitasaka, H. Natori and H. Takabatake "On scale invariant features and sequential Monte Carlo sampling for bronchoscope tracking" Proc. SPIE Medical Imaging 2010: Visualization, Image-Guided Procedures, and Modeling

28. Y. Zhou, E. Yeniaras, P. Tsiamyrtzis, N. Tsekos and I. Pavlidis "Collaborative tracking for MRIguided robotic intervention on the beating heart" Proc. Medical Image Computing and ComputerAssisted Intervention, pp. 351-358, 2010

29. O. Cappé , S. J. Godsill and E. Moulines "An overview of existing methods and recent advances in sequential Monte Carlo" Proc. IEEE, vol. 95, no. 5, pp. 899-924, 2007

30. A. Blake and M. Isard Active Contours 1998, Springer-Verlag

31. H. Kadhem, D. Rodriguez, J. Tena , K. Wells, E. Lewis and M. Guy; "Ultra low dose CT attenuation correction maps for emission computed tomography" Proc. IEEE Nuclear Science Symp./Medical Imaging Conf. Rec., pp. 2123-2127, 2006

32. J. Jones, E. Lewis, M. Guy and K. Wells "A virtual dissection based registration to model patientspecific respiratory motion" IProc. IEEE Nuclear Science Symp./Medical Imaging Conf. Rec., pp. 35713576,2009

33. M. Alnowami , E. Lewis, M. Guy and K. Wells "Marker-less tracking for respiratory motion correction in nuclear medicine imaging" Proc. IEEE Nuclear Science Symp./Medical Imaging Conf. Rec., pp. 3118-3121, 2010

34. K. Wells , B. Goswami , A. A. Rahni , J. Jones , M. Alnowami , E. Lewis and M. Guy; "Modeling respiratory motion variations in the 4D NCAT phantom" Proc. IEEE Nuclear Science Symp./Medical Imaging Conf., pp. 2534-2539, 2009

35. A. Neumaier and T. Schneider "Estimation of parameters and eigenmodes of multivariate autogressive models" ACM Trans Math. Softw., vol. 22, no. 1, pp. 27-57, 2001

36. F. Daum "Nonlinear filters: Beyond the Kalman filter" IEEE Aerosp. Electron. Syst. Mag., vol. 20, no. 8, pp. 57-69, 2058

37. M. Turk and A. Pentland "Eigenfaces for recognition" J. Cognit. Neurosci., vol. 3, no. 1, pp. 71-86, 1991

38. N. Otsu "Threshold selection method from gray-level histograms" IEEE Trans. Syst. Man. Cybern., vol. 9, no. 1, 1979

39. P. Jensfelt , O. Wijk, D. Austin and M. Anderson "Experiments on augmenting condensation for mobile robot localization" Proc. IEEE Int. Conf. Robotics and Automation, pp. 2518-2524, 2000

40. J. Jones, E. Lewis, A. A. Rahni , M. Guy , V. Ezhil and K. Wells; "A patient-specific, virtual dissection based registration to reduce breathing motion artefacts" Proc. IEEE Nuclear Science Symp./Medical Imaging Conf., 2011 


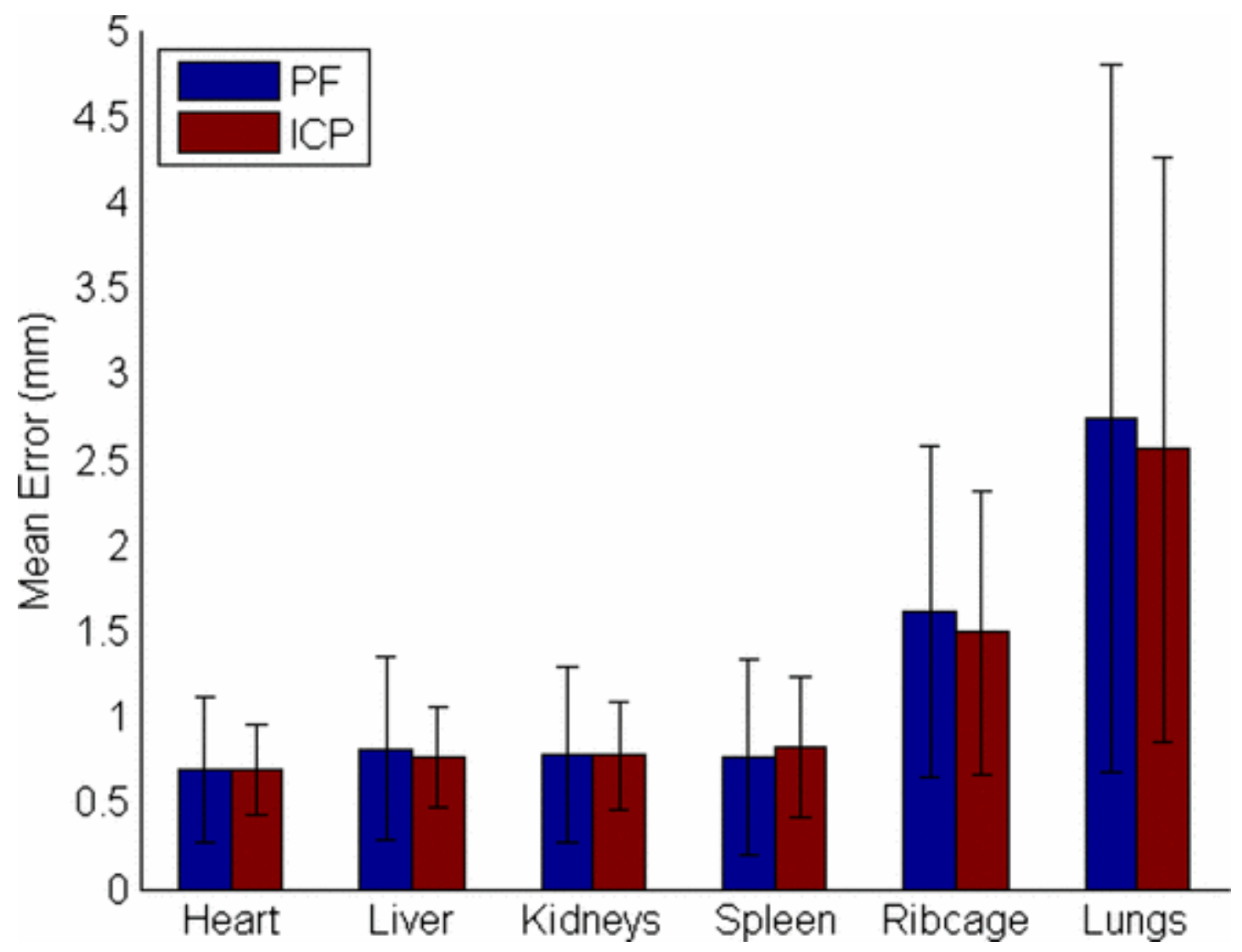

